\title{
MEMRI Characterization and Quantification Maps of Human Pancreatic Islets
}

\author{
Suraj Serai ${ }^{1 *}$, Lara Leoni ${ }^{2}$, Muhammad Haque ${ }^{2}$ and Brian B. Roman ${ }^{1,2}$ \\ *Correspondence: suraj.serai@gmail.com \\ 'Department of Bioengineering, University of Illinois at Chicago. \\ ${ }^{2}$ Department of Radiology, University of Chicago. USA.
}

\begin{abstract}
Recent progress in clinical islet transplantation has accentuated the need for the development of a dependable eans to characterize islet product after it is isolated from a donor pancreas and before it is infused into an islet transplant recipient. Especially the process of optimizing techniques used to determine the quality, functionality, integrity, cellular identity and viability of isolated pancreatic islets by using dynamic micro MR imaging would be valuable to the islet transplantation team. We have developed methods of high resolution Manganese (Mn) enhanced magnetic resonance imaging (MEMRI) for visual documentation of the islet functionality and have attempted to characterize its MR properties.
\end{abstract}

Keywords: Islet transplantation, MEMRI, T1 mapping.

\section{Introduction}

Diabetes mellitus has been inextricably linked to the loss of functionality in the insulin producing islet cells of the exocrine pancreas. Pancreas transplantation is the available therapeutic modality, which can potentially stop the progression of diabetic complications without increasing the incidence of hypoglycemic events [1]. However, this procedure, usually performed simultaneously with a kidney graft, has a high morbidity and a significant mortality rate. In this context, islet transplantation, an emerging therapeutic modality, can be considered as a less invasive endocrine replacement therapy and has the potential to achieve insulin independence and freedom from hypoglycemic attacks $[2,3]$. Relatively new, there are many questions still unresolved which impact the efficacy of this approach. These include isolation regimes, isolation media, number and functional capacity of transplanted islets and their site of engraftment. The further development of endocrine replacement therapy for the treatment of diabetes will depend on the availability of high quality islets and addressing the unresolved issues.

Islets are clusters of cells located in the pancreas. Together, these clusters of cells regulate blood sugar (glucose) levels. Islets comprise only $2-3 \%$ of the pancreas. Beta cells constitute approximately $75 \%$ of the islet cells, generally located within the center of the islet $[3,7]$. They secrete the hormone insulin, which decreases blood sugar levels, and amylin, which modulates the action of insulin. Insulin is secreted from the $\beta$-cells in a biphasic manner when the blood glucose concentration rises above the normal level which is believed to be $\sim 5 \mathrm{mM}$ in humans.Alpha cells constitute approximately $20 \%$ of the islet, generally located around the periphery of the islet.

They secrete the hormone glucagon, which serve to increase blood sugar levels.Delta cells constitute approximately $5 \%$ of the islet cells, generally located around the periphery of the islet. They are neurosecretory cells which release the hormone somatostatin, a paracrine inhibitor of insulin secretion [2]. If the function of human islets can be visualized and quantified by imaging methods, it may be possible to evaluate different islet isolation and transplantation regimes for effectiveness of engraftment. However because they have been less than 400 Microns in average and constitute less than $2 \%$ of the adult pancreas, islet cells have been traditionally difficult to image invivo and yield relatively few specimens per procedure for invitro evaluation $[\mathbf{2}, \mathbf{7}]$.

Contrast MRI provides information on sample properties by assessing parameters - such as spin density, susceptibility, molecular motions resulting from diffusion and perfusion, and $\mathrm{T} 1$ and $\mathrm{T} 2$ relaxation times.Preferential enhancement of signal intensity can be obtained by selectively altering these relaxation parameters [5]. In order to fully appreciate any techniques which take advantage of the manipulation of these parameters it is critical to make quantitative measurements of them under controlled and well characterized treatment conditions.Additionally, before the study of any contrast agent effects, T1 and T2 of control and activated islets were needed to be measured to establish baseline values. A non-invasive MRI method to differentiate functional versus non-functional $\beta$-cells is a powerful technique as applied to transplantation,

(C) 2012 Serai et al; licensee Herbert Publications Ltd. This is an open access article distributed under the terms of Creative Commons Attribution License (http://creativecommons.org/licenses/by/3.0),This permits unrestricted use, distribution, and reproduction in any medium, provided the original work is properly cited. 
however such a technique requires optimization and sensitivity to function.

One such technique is Mn enhanced MRI (MEMRI). Using this technique, functional $\beta$-cells take-up $\mathrm{Mn}$ ions through the calcium channels and enhance their MR signal intensity compared to non-functional cells $[10,12,16,17,18,24,28]$. Manganese is a mineral element that is both nutritionally essential, useful in a number of physiologic processes and potentially toxic. In its worst form via inhalation, manganese toxicity can result in a permanent neurological disorder with symptoms similar to those of Parkinson's disease, including tremors, difficulty walking, and facial muscle spasms $[4,20]$. Although concerns arise about Mn toxicity as a contrast agent in human pancreatic islets, the doses examined did not exhibit negative effects such as cell lyses or hampered insulin secretion. To get most out of this technique, data were obtained to establish acquisition parameters for MR image acquisition of activated versus non-activated human pancreatic islets. Analysis of control and activated islets demonstrates the presence of both intra- and inter-islet characteristics which will be key in the interpretation of functional data in vivo.

The variation of induced magnetization in biological tissues is the source of image contrast. The most basic contrast generating mechanisms are based on spin density, diffusion, $\mathrm{T} 1$ and $\mathrm{T} 2$ difference between tissues [5]. Contrast agents reduce the $T 1$ relaxation of the target tissues and increased the contrast while the signal from non-target tissues remains the same known as T1-weighted. Commonly used MR contrast agents are $\mathrm{Fe}^{+3}, \mathrm{Mn}^{+2}$, and $\mathrm{Gd}^{+3}$ ions.

$\mathrm{Mn}$ has an ionic radius close to that of Calcium and is believed to be handled similarly in many biological systems. Divalent Manganese ions $\left(\mathrm{Mn}^{+2}\right)$ ions are paramagnetic and can enter cells through voltage gated calcium channels $[11,12,17]$. $\mathrm{Mn}^{+2}$ accumulations in $\beta$-cells due to Glucose Stimulation should alter T1 relaxation times offering an approach to sensitize MRI to calcium influx in the pancreas (Figure 1). $\left(\mathrm{Mn}^{+2}\right)$ as a contrast agent reduces the $\mathrm{T} 1$ relaxation of the target tissues and increases the signal while the signal from the background remains the same as $T 1$ weighted. The increase in signal enhancement is proportional to the concentration of the contrast agent. We have shown previously methods to obtain high resolution imaging of islets $[11,12,14,17,18,23]$. This study focuses on assessing human pancreatic islets and characterizing their MR properties.

\section{Material and Methods}

Islets

Human islets, isolated from cadaveric donors were obtained from the Department of Surgery at the University of Illinois at Chicago in accordance with the University of Illinois and the University of Chicago IRB protocols. Each batch of islets was from a different donor, however for our experiments, 35 to 40 different batches were used for multiple experiments

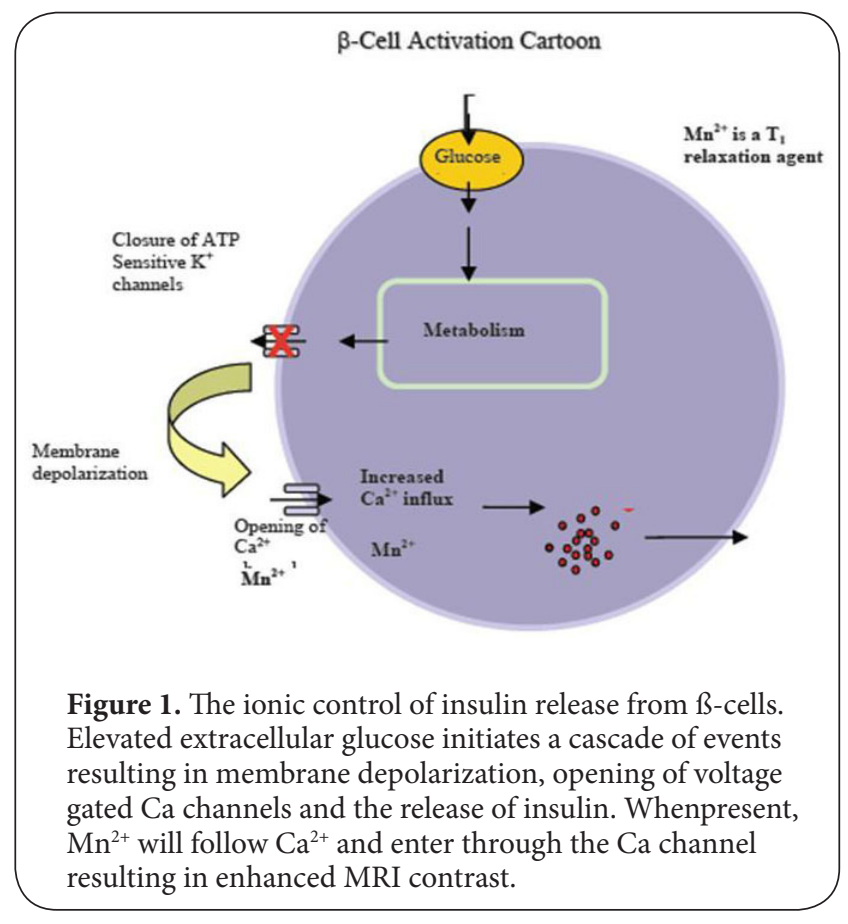

and validation. Islet purity, viability and functionality were assessed for different batches prior to all experiments. Care was taken to include islets that were within $10 \%$ range of purity, viability and functionality to reduce intra-batch and inter-batch variability. They were incubated with final wash solution supplemented with Insulin-Transferrin-Selenium and Pen/Strep for 24 hours after isolation and in certain cases were hand-picked to reduce impurities.

\section{Non-stimulated Islets}

Islets were first incubated in Krebs Ringer Buffer (KRB) solution at $1.67 \mathrm{mM}$ glucose for 30 minutes. Islets were then rinsed three times with KRB and control islets were loaded into $2 \mathrm{~cm}$ long micro capillary tube of $570 \mu \mathrm{m} \mathrm{ID.}$

\section{Mn treated Stimulated \& Unstimulated Islets}

Islets were switched to KRB solution with 35,50 or $100 \mu \mathrm{M}$ $\mathrm{Mn}$ as needed along with $16.7 \mathrm{mM}$ for glucose stimulation or $1.67 \mathrm{mM}$ for unstimulated sample for 30 minutes followed by rinsing. The viability of islets was confirmed using standard trypan blue exclusion assay before and after imaging [26].

\section{Streptozotocin (STZ) treated Islets}

STZ is a naturally occurring chemical that is particularly toxic to the insulin-producing beta cells of the pancreas in mammals $[6,9] .40 .5 \mathrm{mgs}$ STZ was dissolved in $1 \mathrm{ml}$ of freshly prepared Sodium Citrate buffer. $80 \mu \mathrm{L}$ of STZ-citrate solution was added to $2 \mathrm{ml}$ of KRB buffer to obtain the required STZ concentration of $2.2 \mathrm{mM}$. The islets were incubated in this solution for 30 minutes. The viability of islets was confirmed using trypan blue exclusion assay before and after imaging. 


\section{Sample Preparation}

Prior to imaging, the islets were incubated in KRB solution. The islets were pipetted into sample glass tubes for imaging. The sample tubes were mounted on a home built double loop Archimedes spiral surface coil (OD of $750 \mu \mathrm{m}$ ) assembly and inserted into the Bruker Micro 5 Imaging Probe (triple axes gradients of maximum strength 2000 gauss $/ \mathrm{cm}$ ). The assembly is interfaced with the microimaging probe in such a way that the tune and match components and the leads are placed on a specifically designed holder that can be integrated with the surface coil. The surface coil can then be aligned perpendicular to the uniform static field and the linear gradient field.

\section{MR Instrumentation}

All experiments were conducted in a $56-\mathrm{mm}$ vertical bore 11.7 T magnet using a Bruker DRX Avance Spectrometer (Bruker, Billerica, MA). Spin echo (SE) sequences were used to obtain spin density, T1 and T2 weighted contrast images. The typical imaging parameters were: $\mathrm{TE}=8 \mathrm{~ms}$, $\mathrm{TR}=500 \mathrm{~ms}, \mathrm{Mx}=256, \mathrm{NEX}=15$, Slice thickness $=0.3$ $\mathrm{mm}, \mathrm{FOV}=0.3 \mathrm{~mm}$, In-plane resolution $=10 \mu \mathrm{m}$. The MR images acquired were viewed and processed using ImageJ (National Institutes of Health, USA). T1 \& T2 measurements were performed, exponential equations were used to fit the curve between the recovery time and intensity, and $\mathrm{T} 1$ and T2 relaxation maps produced using Matlab (The Mathworks Inc, Natick, MA).

\section{T1 and T2 measurements}

The Longitudinal relaxation time (T1) was calculated by saturation recovery method using multi slice multi echo pulse sequence. Fourteen experiments were acquired with different relaxation time ranging from $100 \mathrm{msec}$ through $2400 \mathrm{msec}$. Other imaging parameters include matrix size $=128, \mathrm{TE}=7.96 \mathrm{msec}$. , FOV $=0.30 \mathrm{~mm}$, Slice thickness $=$ $0.30 \mathrm{~mm}, \mathrm{NEX}=10$.The respective $\mathrm{T} 1$ and $\mathrm{T} 2$ relaxation times were calculated. computer generated $\mathrm{T} 1$ map using MATLAB (Natick, MA) provides us the ability to calculate T1 pixel by pixel.

Transverse relaxation (T2) was calculated using CarrPurcell Mieboom-Gill (CPMG) pulse sequence with equally spaced 32 echoes. T2 relaxation time was calculated using Bloch equation by plotting signal intensity versus the echo time.

Exponential equations were used to fit the curve between the recovery time and intensity, and $\mathrm{T} 1$ and $\mathrm{T} 2$ relaxation maps produced using Matlab (The Mathworks Inc, Natick, $M A)$.

\section{In-vivo imaging and set-up}

All surgical procedures were performed in accordance to the approved University of Chicago and University of Illinois at Chicago animal care protocols. Rats used in the procedure were anesthetized with $1.5 \%$ isoflurane along

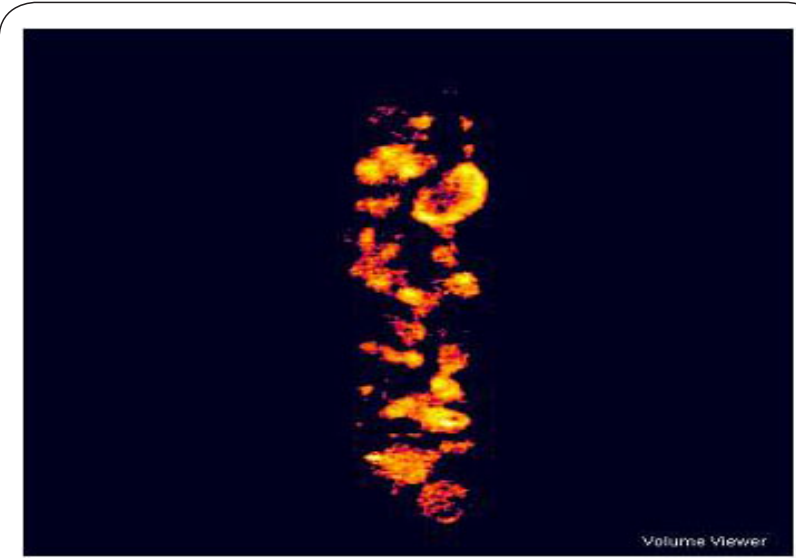

Figure 2. MR Image of Human Pancreatic Islets. (Twodimensional high resolution coronal image of isletsin a glass capillary tube. $\mathrm{Mx}=256 \mathrm{x} 256, \mathrm{TE}=12.56 \mathrm{~ms}, \mathrm{TR}=800 \mathrm{~ms}$, NEX $=250$, Resolution $=10$ microns)

with oxygen. The animal was kept warm with hot air flowing on him. ECG and body temperature were monitored with probes connected using SA Instrument (Stony Brook, NY, USA). Animal was continuously monitored for heart rate and respiration.

Coronal images of the pancreas were obtained using both spin (MSME) and gradient echo (FLASH, MPRAGE) to obtain heavily $T 1$ weighted images. Typical imaging protocol for MSME was TE $=9.4 \mathrm{~ms}, \mathrm{TR}=350 \mathrm{~ms}$, matrix = $128 \times 128, \mathrm{FOV}=5.8 \times 6.1 \mathrm{~cm}, \mathrm{NEX}=8$, Resolution $=429$ $x 429$ micron; FLASH, $\mathrm{TE}=4.2 \mathrm{~ms}, \mathrm{TR}=108 \mathrm{~ms}, \mathrm{FA}=60$, $\mathrm{NEX}=8$, Matrix $=192 \times 192$, resolution $286 \times 286$ micron, MPRAGE, TR $=10.7 \mathrm{~ms}, \mathrm{TE}=3.3 \mathrm{~ms}$, No. of Segments $=4$, Segment duration $=340 \mathrm{~ms}, \mathrm{FA}=10$, Inversion delay $=$ $750 \mathrm{~ms}, \mathrm{NEX}=6$, matrix $=128 \times 128$. MR imaging post $\mathrm{Mn}$ infusion was with $75 \mathrm{mM}$ Manganese Chloride solution.

\section{Results}

Figure 2 illustrates a 2D high resolution spin density MR image of human islets (neither glucose stimulated nor $\mathrm{Mn}$ treated). The T1 values of islets are in the range of 500-700 msec compared to the surrounding media T1 of $\sim 1150-2000$ msec (Figure 3). The T2 values from the islets are in the range of $\sim 19-39 \mathrm{msec}$ compared to the surrounding media T2 $<14 \mathrm{msec}$ (Figure 4).

To determine if the glucose stimulated uptake of $\mathrm{Mn}$ could be seen in human islets, a similar protocol developed by our group for use with rodent islets was implemented [10].The viability of islets was confirmed using trypan blue before and after imaging. Figure $5 \mathrm{~A}$ is a high resolution $2 \mathrm{D}$ MR image of control islets (left) and stimulated islets (right) that have been glucose activated in the presence of $\mathrm{Mn}$. Contrast was achieved for $\mathrm{Mn}$ concentrations ranging between $35 \mu \mathrm{M}$ and $100 \mu \mathrm{M}$. As opposed to our previous work with rodent islets for which highest contrast was achieved between $2.5 \mu \mathrm{M}$ to $25 \mu \mathrm{M}$, no contrast was seen 


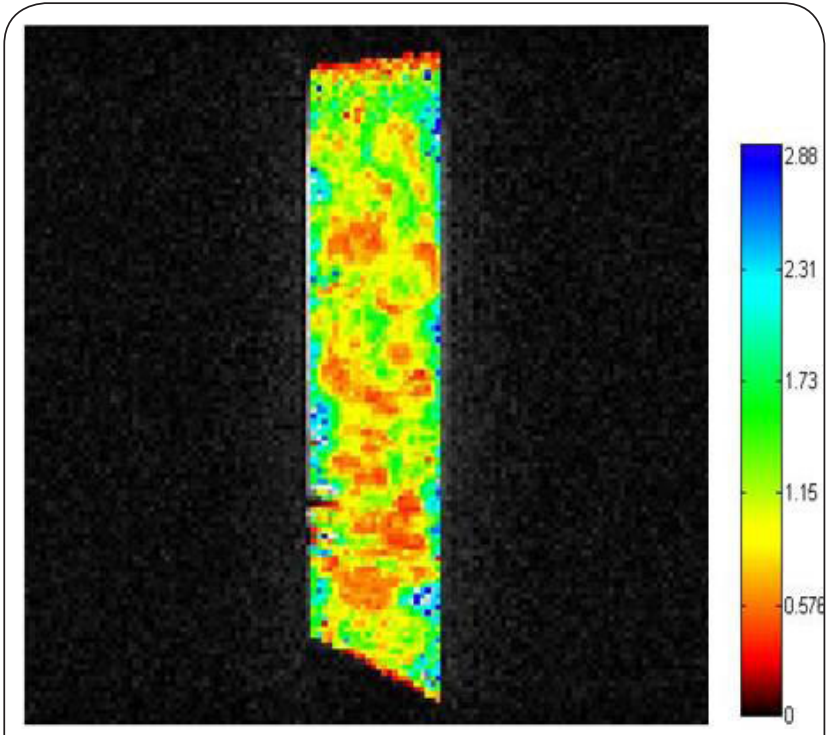

Figure 3. T1 map of islets (Matrix size $=128 \times 128, \mathrm{E}=7.95$ $\mathrm{ms}$, TR ranges from 100 to $3000 \mathrm{~ms}, \mathrm{NEX}=10$.

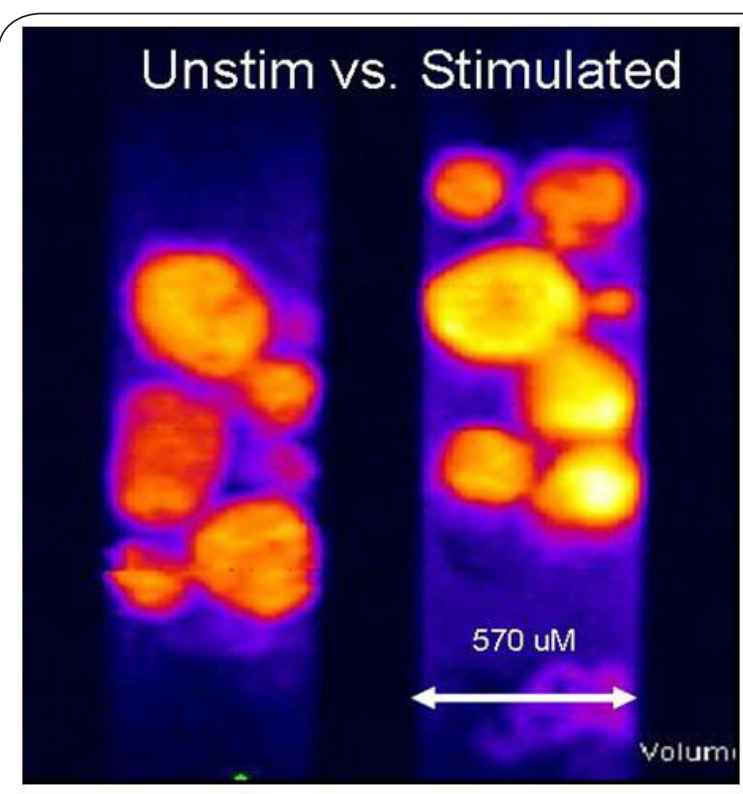

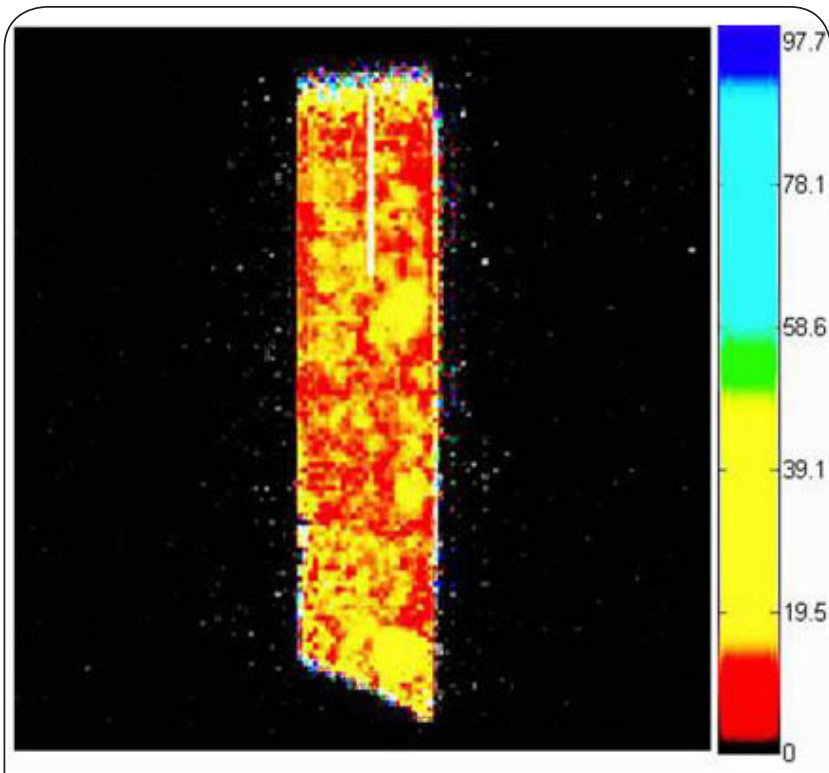

Figure 4. T2 map of islets. (Matrix Size $=128 \times 128, \mathrm{TE}=7.95$ ms, No. of Echoes $=32$, TR=3000 ms, =10.)

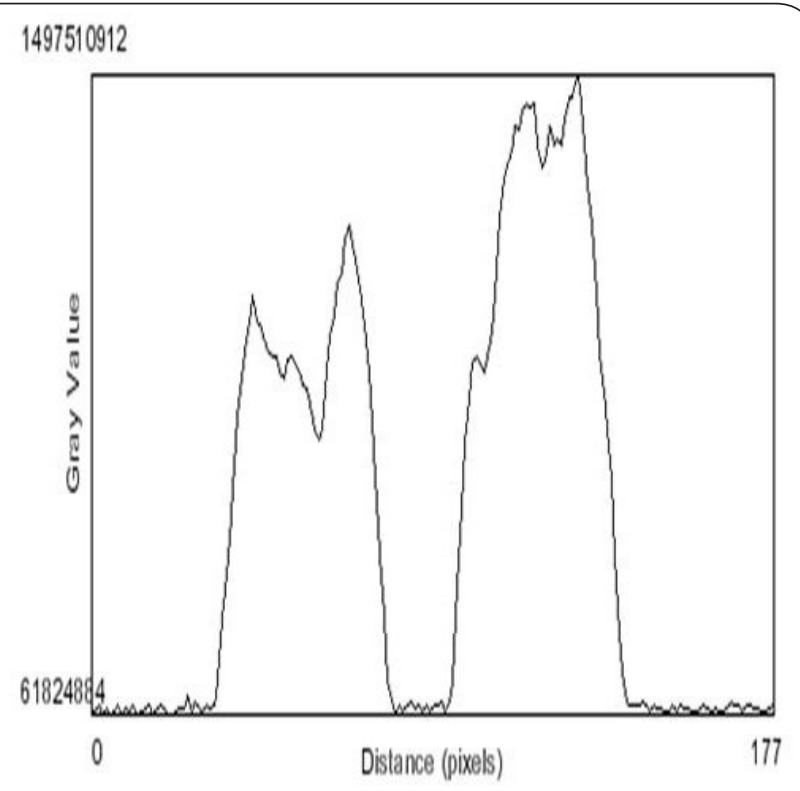

Figure 5. Control versus Stimulated Islets. (A) High resolution 2D image of control (left) \& stimulated (right) human islets. Islets were exposed to $35 \mu \mathrm{M} \mathrm{Mn} \& 2 \mathrm{mM}$ (left) \& $16.7 \mathrm{mM}$ (right) glucose. (B) Line profile of the signal intensity across the two tube samples illustrates intra- and inter-islet characteristics.

at lower Mn concentrations. However, despite the higher Mn concentrations employed, in this case as well no toxic effects on insulin secretion were observed. The activated islets show a pronounced increase in contrast due to the influx of $\mathrm{Mn}$ and a resulting change in $\mathrm{T} 1$ relaxation. A line profile across the two sample tubes shown in Figure 5B illustrates the intra-islet signal intensity variation and the inter-islet difference in contrast due to glucose stimulation in the presence of $\mathrm{Mn}$. The intra-islet distribution is probably due to the distribution of $\beta$-cells inside the islets.

Post $\mathrm{Mn}$ and glucose uptake, The $\mathrm{T} 1$ values were reduced by approx. $20-30 \%$ along with a similar increase in signal to noise ratio. The $\mathrm{T} 1$ generated map and the measured SNR quantify this data. Although concerns exist about $\mathrm{Mn}$ toxicity, the doses employed did not exhibit any acute negative effects; islets exposed to Mn showed normal glucose sensitivity and insulin secretion which was verified by ELISA assay tests and trypan blue viability studies. An 


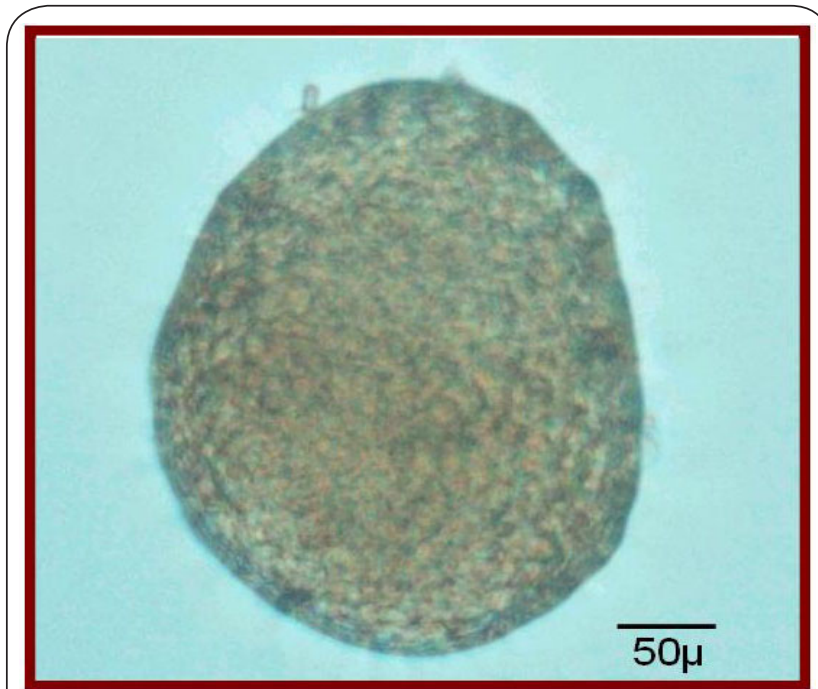

Figure 6. Islet stained with trypan blue for viability: imulated islet - incubated with high glucose and .25mM Mn dose.

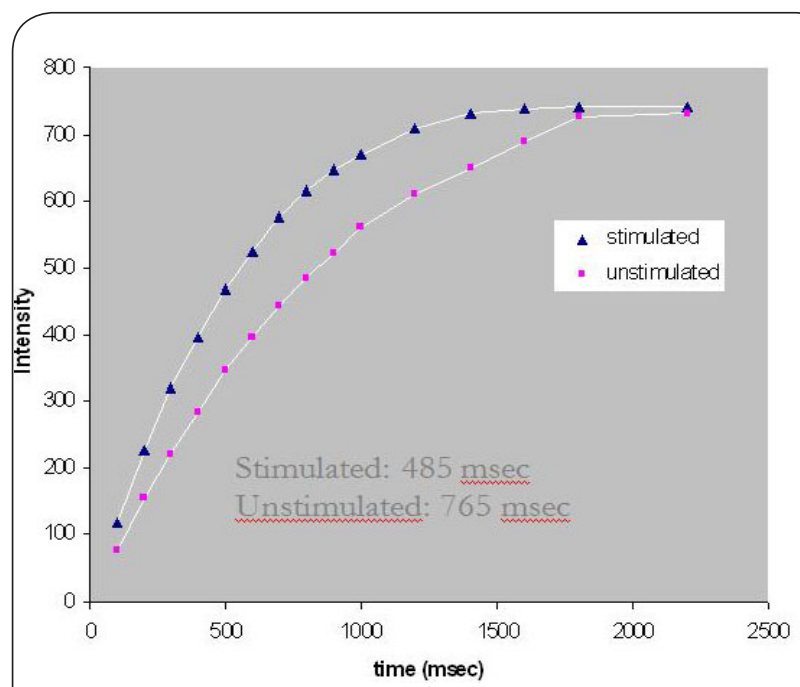

Figure 7. T1 Plot. T1 plot of control (left) and stimulated human islets (right) supports the data of reduced T1 due to Mn uptake.

example image of an islet stained with typan blue, current test certainly considered to make it possible to see for viability is shown in Figure 6 . The live cell does not take up the dark trypan blue stain and the cell membrane appears intact.

A signal to noise improvement of $30 \%$ was obtained with islets incubated with $100 \mu \mathrm{M} \mathrm{Mn}$ for 30 mins and similar results were obtained for islets incubated with $50 \mu \mathrm{M} \mathrm{Mn}$ for 45 mins. Islets treated with STZ were found to exhibit no increase in SNR and hence were determined non-functional.

A T1 comparison plot of stimulated and unstimulated islets in presence of $\mathrm{Mn}$ is shown in Figure 7.A T1 relaxation map,

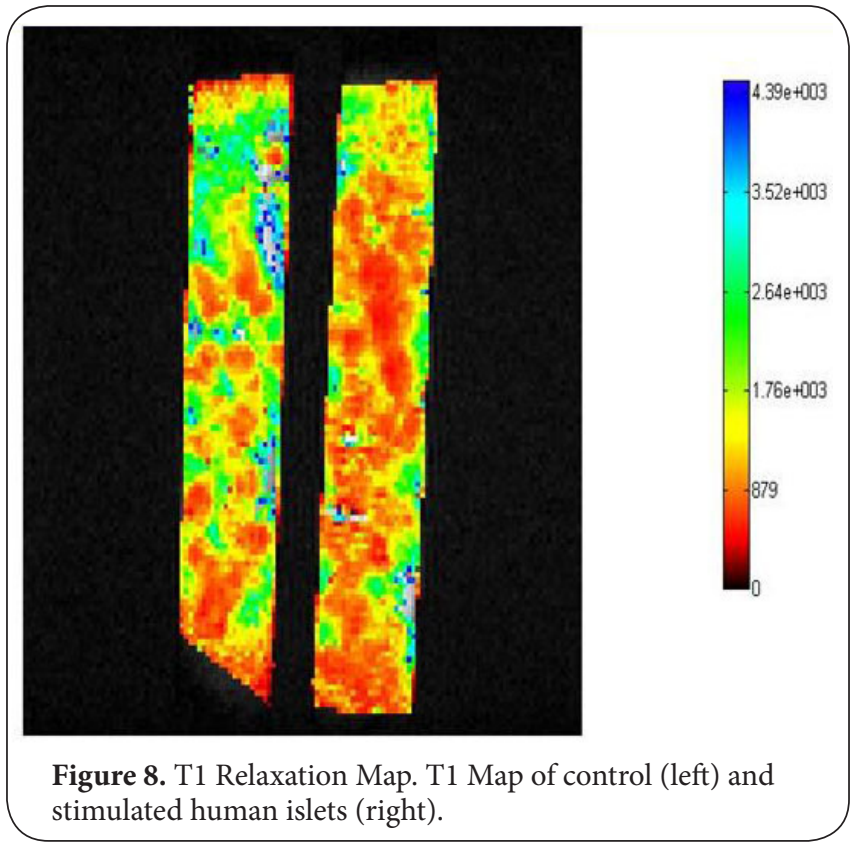

comparing stimulated and unstimulated islets, generated using MATLAB obtained is shown in Figure 8. T1 value of stimulated islet is observed to be in the order of 400 to $500 \mathrm{msec}$ (std. dev of +/- $50 \mathrm{msec}$ ) with, while the values for control islets stand between 700 to $800 \mathrm{msec}$ (std. dev of $+/-50 \mathrm{msec}$ ).

In-vivo Imaging: The rat pancreas is a diffused organ in the cranial abdominal cavity and virtually has no defined morphology. It is a gelatin-like mass embedded in mesentery tissues originating from the duodenum, extending along the stomach, and connecting to the spleen at one end and other end buried in the intestinal region. With the help of precise animal set up, we have been able to accurately track down the pancreas and observe its $\mathrm{Mn}$ uptake in the presence of glucose activation. Figure $\mathbf{9}(\mathbf{a}, \mathbf{b} \mathbf{\&} \mathbf{c})$ shows the success of our desired application.Our data confirm that Mn-enhanced MRI (MEMRI) used in combination with high resolution $\mathrm{MRI}$ is a promising technique to non-invasively assess the functionality of human islets with the in-vivo rodent pancreas model.

\section{Conclusion}

MEMRI was used to successfully present a promising technique to non-invasively assess the functionality of human islets. Careful MR characterization of isolated human islets has been performed which should result in further optimization of the imaging technique when applied in vivo. Quantification and understanding of $\mathrm{T} 1$ and $\mathrm{T} 2$ changes in the islets as a baseline measurement is critical to future functional islet imaging. our results demonstrate the feasibility of obtaining high-resolution MR images and activation maps of isolated human pancreatic islets. T1 \& T2 characterization of human islets under control conditions were successfully achieved. The concentration 

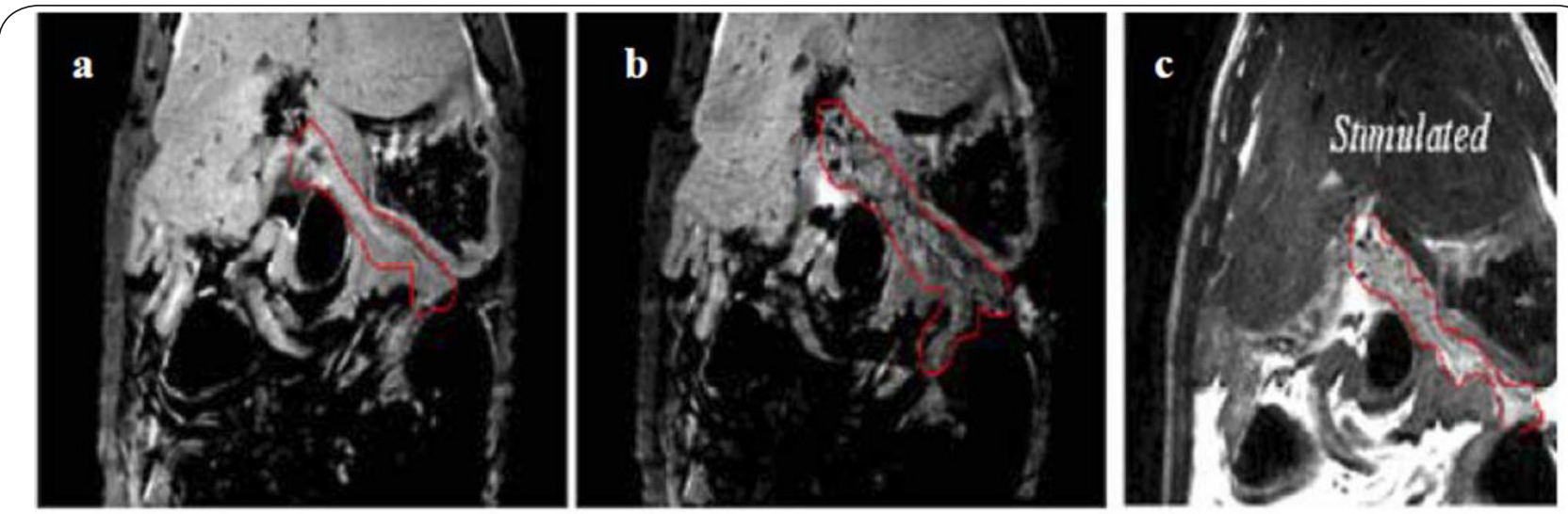

Figure 9. Invasive MEMRI of rat pancreas. (a) Pre Mn pre glucose, (b) post Mn and (c) post Mn and glucose infusion.

of $\mathrm{Mn}$ required for image enhancement has been shown not to inhibit human islet function and provide data on intra- and inter-islet characteristics. T1 values of islets at various stages were measured and has been found to be $700-900 \mathrm{msec}$ and with Mn enhancement the value reduces to 400 to $600 \mathrm{msec}$ range. The T1 values varies from batch to batch and this variation is mainly due to viability, islet heterogenity, incubation times and isolation techniques.

$\mathrm{T} 2$ values from the islets are in the range of $22-35 \mathrm{msec}$ with no pronounced change by treatment with $\mathrm{Mn}$. It is our observation that freshly isolated islets respond well to the Mn induced T1 \& T2 changes. Beyond proof-ofconcept, careful MR characterization of isolated human islets has been performed which should result in further optimization of the imaging technique when applied in vivo pre and post transplantation. The availability of a noninvasive technique capable of monitoring the functionality of islets/ $\beta$-cells in vivo will enhance the understanding of diabetes and facilitate its treatment.

\section{Discussion}

In a quest to find a cure for Diabetes, several researchers across the globe have tried and published results of pancreas and islet transplantation experimental trials. Receiving pancreas and/or kidney transplantations, results in the use of high immunosuppressive drugs, which also increase the body-toxicity levels. American Diabetes Association recommends that pancreas transplantation should only be considered as an adjunct to kidney transplantation $[1,15]$. In this context, islet transplantation, an emerging therapy since 2000 , could be considered as a minimally invasive replacement therapy and can result in insulin independence and freedom from hypoglycemic attacks $[22,27]$. The further development of endocrine replacement therapy for the treatment of diabetes depend on the availability of high quality islets and addressing unresolved issues such as functional islet mass engraftment rate, cell apoptosis, and immunorejection.

Current islet isolation procedures have been shown to cause damage to both the islet as a microdomain and to single islet cells. A significant proportion of the isolated tissue consists of fragmented islets lacking basement membrane and whose peripheral cells have severe plasma membrane damage. The integrity of the islets is fundamental to their in vivo performance and different techniques have been used to score human pancreatic islet preparations prior to transplantation $[3,19,21]$.

Currently existing methods to test human islet function include transplantation in immunodeficient mice, assessment of insulin biosynthesis, in vitro perifusion glucose static incubation $(\mathbf{8}, \mathbf{1 3}, \mathbf{2 5})$. Correction of hyperglycemia in an animal model is the best and the most accurate assessment but this needs a large number of islets and could take several days for an outcome. Of the two mentioned in vitro techniques, glucose static incubation is the fastest and easiest way to obtain an insulin secretory activity index but these methods are prone to errors and most often also results in scattered statistical results. In addition, this measurement gives an idea of the amount of insulin that acts on the tissues, but cannot add information on the dynamics of the hormone in terms of secretion and clearance. We have developed methods of high-resolution dynamic perfusion based MR imaging techniques for visual documentation of the islet functionality and to determine cell viability. A similar imaging paradigm used earlier for rodent islet activation (MEMRI) is also used as tool to image $\beta$-cell functionality in isolated human pancreatic islets $[11,17]$. T1 measurement curves and SNR values as an outcome of this proposed technique could be used as standard assessment values in addition to the routinely performed tests to provide a more detailed analysis and determine the quality and functionality by Mn uptake; integrity, cellular identity and viability by staining techniques; and insulin secretion by fraction analysis of isolated pancreatic islets by using this dynamic micro MR imaging. This can lead to higher success rates of islet transplantation results by choosing the right batch of islets.

The islets used in our experimental technique can 
also be and were recovered back for additional lab tests including histological assessments and viability testing.We hope that our expertise in this area will lead to universal acceptance of objective, standardized methods of assessing islet functionality and quality in the beta cell replacement therapy world.

Our proposed technique can work as a standard assessment in addition to the routinely performed tests to provide a more detailed analysis and determine the quality, functionality, integrity, cellular identity and viability of isolated pancreatic islets by using this dynamic micro MR imaging. However, with the current set-up, labs having facilities and regular access to MR micro-imaging system can only apply this method to a regular and effective use.

Correction of hyperglycemia in an animal model is the best and the most accurate assessment of the proposed and developed technique of $\mathrm{Mn}$ uptake and contrast development by functional pancreatic islet cells. Although many advances have been made in the isolation and transplantation techniques, the factors that determine the location, function and rejection of these grafts are not clearly understood. This lack of information is primarily due to lack of techniques available for such assessments. Normal clinical MRI techniques currently implemented are sensitive to pancreatic lesions and tumors at high spatial resolution but do not offer significant functional information.

Our developed technique is a simple to design, develop and can be used as a pre-assessment in the determination of islet functionality. High resolution functional MR imaging of islets as an extension of molecular imaging providing islet metabolism has been demonstrated. The subtracted image shows a clear indication of increased signal intensity in stimulated versus unstimulated islets. Despite the encouraging results, much animal work is still required before this project can be taken closer to its clinical application. The isolated human islets can be transplanted in diabetic nude mice at pre-decided sites, i.e. under the kidney capsule, intra-peritoneal or on the hepatic regions. Mice/ Rodents can be made diabetic by way of streptozotocin injection by tail IV under anesthesia and then subjected to islet transplantation to regulate hyperglycemia.Increased glucose in the presence of $\mathrm{Mn}$ would activate the transplanted islets and the graft region will demonstrate a change in MR signal intensity compared to non-activated cells.

\section{Competing interests}

The authors declare that they have no competing interests. Acknowledgements

This work was done as part of graduate research by Suraj Serai at Department of Bioengineering, University of Illinois, Chicago. Graduate Research Advisors: Brian Roman, Ph.D., Richard Magin, Ph.D., Lara Leoni, PhD., and Robert Kleps, Ph.D. A National Institute of Health grant R01EB001828 to BBR supported this work. We also thank Dr Jose Oberholzer and his laboratory (Department of Surgery, Univ. of Illinois, Chicago) for helpful discussions and for providing islets.

\section{Publication history}

Editor: Jon S. Odorico, University of Wisconsin-Madison, USA.

Received: 24-Oct-2012 Revised: 27-Nov-2012

Reviwed: 30-Nov-2012 Accepted: 12-Dec-2012

Published: 19-Dec-2012

\section{References}

1. Beisswenger PJ. Type 1 diabetes. In: Leahy JL, Clark NG, Cefalu WT, eds. Medical Management of Diabetes Mellitus. New York: Marcel Dekker Inc; 2000:95-113.

2. M Brissova, MJ Fowler, WE Nicholson, A Chu, B Hirshberg, DM Harlan and AC Powers: Assessment of human pancreatic islet architecture and composition by laser scanning confocal microscopy. Journal of Histochemistry \& Cytochemistry 2005, 53:1087-97. | Article | PubMed

3. MJ Barnett, D McGhee-Wilson, AM Shapiro and JR Lakey: Variation in human islet viability based on different membrane integrity stains. Cell Transplant 2004, 13:481-8. I PubMed

4. TW Clarkson: Metal toxicity in the central nervous system. Environmental health perspectives 1987, 75:59-64. I PubMed Abstract | PubMed Full Text

5. R Damadian: Tumor detection by nuclear magnetic resonance. Science 1971, 171:1151-3. I Article I PubMed

6. DL Eizirik, S Sandler, N Welsh and C Hellerstrom: Preferential reduction of insulin production in mouse pancreatic islets maintained in culture after streptozotocin exposure. Endocrinology 1988, 122:1242-9. | Article | PubMed

7. DL Eizirik, DG Pipeleers, Z Ling, N Welsh, C Hellerstrom and A Andersson: Major species differences between humans and rodents in the susceptibility to pancreatic beta-cell injury. Proc Natl Acad Sci U S A 1994, 91:9253-6. | PubMed Abstract | PubMed Full Text

8. Rorsman Patrik. Insulin secretion: Function and therapy of pancreatic beta-cells in diabetes. Br J Diabetes Vasc Dis 2005,5:187191.

9. D Dufrane, M van Steenberghe, Y Guiot, RM Goebbels, A Saliez and P Gianello: Streptozotocin-induced diabetes in large animals (pigs/primates): role of GLUT2 transporter and beta-cell plasticity. Transplantation 2006, 81:36-45. | PubMed

10. NV Evgenov, Z Medarova, G Dai, S Bonner-Weir and A Moore: In vivo imaging of islet transplantation. Nat Med 2006, 12:144-8. | Article | PubMed

11. Gimi B, Eroglu S, Leoni L, Desai TA, Magin RL, Roman BB: NMR Spiral Surface Microcoils: Applications. Magnetic Resonance Engineering 2003, 18B:1-8. | Article

12. B Gimi, L Leoni, J Oberholzer, M Braun, J Avila, Y Wang, T Desai, LH Philipson, RL Magin and BB Roman: Functional MR microimaging of pancreatic beta-cell activation. Cell Transplant 2006, 15:195-203. | PubMed

13. M de Groot, BJ de Haan, PP Keizer, TA Schuurs, R van Schilfgaarde and HG Leuvenink: Rat islet isolation yield and function are donor strain dependent. Lab Anim 2004, 38:200-6. | Article | PubMed

14. Haque M, Serai S, Leoni L, Oberholzer J, Honeychuck RV, Roman BB Diffusion Weighted $\mu-M R I$ of Isolated Human Pancreatic Islets. 14 ISMRM, Seattle, Washington, 2005. I PDF

15. D Jirak, J Kriz, V Herynek, B Andersson, P Girman, M Burian, F Saudek and $\mathrm{M}$ Hajek: MRI of transplanted pancreatic islets. Magn Reson Med 2004, 52:1228-33. | Article I PubMed 
Serai et al. Journal of Diabetes Research \& Clinical Metabolism 2012, http://www.hoajonline.com/journals/pdf/2050-0866-1-21.pdf

16. I Komoto, M Kato, A Itami, Y Shimada, R Doi, R Hosotani and M Imamura: Expression and function of the calcium-sensing receptor in pancreatic islets and insulinoma cells. Pancreas 2003, 26:178-84. I PubMed

17. L Leoni, SD Serai, ME Haque, RL Magin and BB Roman: Functional MRI characterization of isolated human islet activation. NMR Biomed 2010, 23:1158-65. | Article | PubMed

18. Leoni L, Serai S, Haque M, Oberholzer J, Magin RL, Roman BB. MEMRI of Glucose Activated Human Pancreatic Islets. 14th ISMRM, Seattle, Washington 2005, I PDF

19. R Lehmann, RA Zuellig, P Kugelmeier, PB Baenninger, W Moritz, A Perren, PA Clavien, M Weber and GA Spinas: Superiority of small islets in human islet transplantation. Diabetes 2007, 56:594-603. I Article I PubMed

20. BS Levy and WJ Nassetta: Neurologic effects of manganese in humans: a review. Int J Occup Environ Health 2003, 9:153-63, I Article I PubMed

21. S Morini, M Braun, P Onori, L Cicalese, G Elias, E Gaudio and C Rastellini: Morphological changes of isolated rat pancreatic islets: a structural, ultrastructural and morphometric study. J Anat 2006, 209:381-92. | Article | PubMed Abstract | PubMed Full Text

22. EA Ryan, JR Lakey, BW Paty, S Imes, GS Korbutt, NM Kneteman, D Bigam, RV Rajotte and AM Shapiro: Successful islet transplantation: continued insulin reserve provides long-term glycemic control. Diabetes 2002, 51:2148-57. | Article | PubMed

23. Serai S, Leoni L, Haque M, Oberholzer J, Magin RL, Roman BB. MR Characterization of Isolated Human Pancreatic Islets. 2005,14 ISMRM, Seattle, Washington. I PDF

24. AC Silva, JH Lee, I Aoki and AP Koretsky: Manganese-enhanced magnetic resonance imaging (MEMRI): methodological and practical considerations. NMR Biomed 2004, 17:532-43. | Article | PubMed

25. M Skau, B Pakkenberg, K Buschard and T Bock: Linear correlation between the total islet mass and the volume-weighted mean islet volume. Diabetes 2001, 50:1763-70. | Article | PubMed

26. W Strober: Trypan blue exclusion test of cell viability. Curr Protoc Immunol 2001, Appendix 3:Appendix 3B. | Article I PubMed

27. $T$ Titus, L Badet and DW Gray: Islet cell transplantation for insulindependant diabetes mellitus: perspectives from the present and prospects for the future. Expert Rev Mol Med 2000, 2:1-28. | Article I PubMed

28. Y Usachev and A Verkhratsky: IBMX induces calcium release from intracellular stores in rat sensory neurones. Cell Calcium 1995,

17:197-206. | Article | PubMed 